\title{
Analisi di una lirica pavesiana: La notte
}

\section{Luciano Vitacolonna}

Università degli Sudi «G. d'Annunzio» di Chieti e Pescara vitaco@libero.it

\section{Abstract}

Quest'articolo presenta una analisi — di tipo sostanzialmente strutturalista — della poesia La notte di Cesare Pavese. Vengono così messi in luce gli aspetti metrico-prosodici, formali e simbolici della lirica, evidenziando soprattutto come le numerose ripetizioni e opposizioni che caratterizzano il testo assumano una valenza connotativa e metaforica.

Parole chiavi: collina; finestra; opposizioni; ripetizioni; simbolismo.

\section{Abstract}

This article presents a structural analysis of Pavese's poem La notte (The night). So the prosodic, formal and symbolic aspects of the lyric are pointed out. The analysis also shows how the frequent reiterations and oppositions that characterize the text take a connotative and metaphorical value.

Keywords: Hill; Window; Oppositions; Reiterations; Symbolism. 
1.

La notte, scritta nell'aprile del 1938, chiude la prima sezione (intitolata Antenati) di Lavorare stanca. E' un componimento, come rileva Tondo (1988: 64), "di una purezza elegiaca straordinaria», il cui linguaggio contrasta con quello «realistico, crudo e violento» delle poesie che lo precedono. Ecco il testo ${ }^{1}$ :

\section{La notte}

Ma la notte ventosa, la limpida notte che il ricordo sfiorava soltanto, è remota, è un ricordo. Perduta una calma stupita fatta anch'essa di foglie e di nulla. Non resta, di quel tempo di là dai ricordi, che un vago ricordare.

Talvolta ritorna nel giorno nell'immobile luce del giorno d'estate, quel remoto stupore.

il bambino guardava la notte sui colli freschi e neri, e stupiva di trovarli ammassati: $\quad 10$ vaga e limpida immobilità. Fra le foglie che stormivano al buio, apparivano i colli dove tutte le cose del giorno, le coste e le piante e le vigne, eran nitide e morte e la vita era un'altra, di vento, di cielo, e di foglie e di nulla.

Talvolta ritorna nell'immobile calma del giorno il ricordo di quel vivere assorto, nella luce stupita.

La struttura metrico-ritmica, in cui ben riconosciamo il verso lungo pavesiano, è alquanto composita e vi spiccano tre pause forti realizzate mediante tre 'accapo interni' ${ }^{2}$. Ecco le caratteristiche metriche principali:

- tutti i versi sono sciolti; va però notato che: $(a)$ i vv. 9 e 12 rimano mediante la stessa parola («colli»); (b) il v. 12 presenta una sorta di rima interna tra "stormivano" e "apparivano»; $(c)$ si possono individuare almeno due 'rime imperfette' (i) tra "ricordo» e «assorto» (vv. 17 e 18), e (ii) tra «coste» e «morte» (vv. 13 e 14$)^{3}$;

1. Il testo è quello riportato in Cesare PAVESE, Lavorare stanca, Torino: Einaudi, 1968, p. 25.

2. Per il termine 'accapo interno' (da accostare all'antilabé'), cfr. Aldo Menichetti Metrica italiana. Fondamenti metrici, prosodia, rima, Padova: Antenore, 1993, p. 453. Sulla funzione della 'pausa centrale' cfr. Marziano Guglielminetti, «Racconto e canto nella metrica di Pavese», Sigma, 3-4, 1964, pp. 22-33; p. 24.

3. Cfr. anche il rapporto - ma troppo distante per costituire una rima imperfetta - fra «di là» (v. 5) e «immobilità» (v. 13). 
- tutti i versi sono piani;

- la lirica — come già detto — presenta tre 'accapo interni' ai vv. 6, 8 e 16;

- i vv. 1, 2, 3, 4, 5, 6, 7, 9, 11, 12, 13, 14, 15, 16 e 17 sono costituiti ognuno da tredici sillabe; anche in questa poesia, dunque, prevale il famoso verso lungo che Pavese avrebbe scoperto casualmente;

— i vv. 8, 10 e 18 sono costituiti ognuno da quattordici sillabe;

- come vuole il modello ritmico accentuativo di Pavese, tutti i versi di tredici sillabe hanno accenti di $3^{a}, 6^{a}, 9^{a}$ e $12^{a}$, mentre tutti i versi di quattordici sillabe hanno accenti di $3^{\mathrm{a}}, 6^{\mathrm{a}}, 10^{\mathrm{a}}$ e $13^{\mathrm{a}}$.

Questa lirica, diversamente da quello che è stato variamente definito come 'parlato lirico', 'narrazione ritmica', 'lirica prosastica' o - per usare i termini dello stesso Pavese — "frase colorita di "parlato»" (Pavese 1965: 270) o "poesia-racconto» (Pavese 1968: 124), offre, come osserva Forti (1964: 38), «la rappresentazione ritmica di una situazione, di una scena, di una presenza umana raffigurata e parlante, che in certi casi può essere quella del poeta stesso che ha recuperato un momento cruciale e determinante della propria vita, spesso il momento vivo delle irripetibili scoperte dell'infanzia». Sarebbe quasi ovvio, eppur fuorviante, accostare La notte a Notturno (del 1940), che appartiene alla sezione successiva (intitolata Dopo) di Lavorare stanca. Infatti, sempre Forti (1964: 46) nota:

In La notte [...] Pavese ritrova uno stupore originario di canto, una ragione sentimentale tutta portante che volge all'idillio, al grande idillio [...] di tipo leopardiano, dove le cose e la loro musica, la presenza e il ricordo, sono un motivo unico di canto felice e definito [...]; mentre in Notturno [...] si alza liricamente la compiuta fusione fra figura e paesaggio, fra immagine e mitica memoria, fra l'occasione ormai perduta e la seconda vista come stregata di un visionario nutrito peraltro di cose e di realtà.

E un altro confronto si potrebbe azzardare con Mattino, che appartiene sempre alla sezione intitolata Dopo. Questa volta, però, a distanziare le due liriche è una certa retorica di Mattino, una sua troppo evidente raffinatezza (alquanto scoperta anche negli echi letterari) che non solo contrasta col 'parlato lirico' più tipicamente pavesiano, ma addirittura scade nello scontato formale e nel banale simbolico.

In La notte ricorrono immagini, situazioni, simboli o metafore (la collina, la finestra, i ricordi), nonché strutturazioni, che sono tipici di Pavese ${ }^{4}$ e che

4. Cfr. Giorgio BÀrberi Squarotti, "Pavese o la fuga nella metafora», Sigma, 3-4, 1964, pp. 165-188; ID., "Lettura di Lavorare stanca», Il mestiere di scrivere, Cesare Pavese trent'anni dopo, Atti del convegno, Comune di Santo Stefano Belbo,1982, pp. 37-62; Dominique Fernandez, L'échec de Pavese, Paris: Grasset, 1967; M. Forti «Sulla poesia di Pavese», Sigma, 3-4, 1964, pp. 34-48; Marziano Guglielminetti e Giuseppe Zaccaria, Cesare Pavese, Firenze: Le Monnier, 1984; Johannes HösLE, «I miti dell'infanzia», Sigma, 3-4, 1964, pp. 202-216; María de las Nieves Muñız MuÑız, Introduzione a Pavese, Roma-Bari: Laterza, 1992; Michele Tondo, Invito alla lettura di Cesare Pavese, Milano: Mursia, 1988; Gianni Venturi, Cesare Pavese, Firenze: La Nuova Italia, 1982. 
Mauron (1966) non esiterebbe a definire 'metafore ossessive' o 'reti ossessive'. Tuttavia, a ben intendere la lirica, è utile e necessario mettere in evidenza - più (o prima ancora) che le 'sovrapposizioni' di Mauron - tutta una serie di opposizioni ${ }^{5}$ e parallelismi, di corrispondenze contrarie e ripetitive, che assicurano al testo - tanto sul piano formale quanto su quello contenutistico - una coesione e una compattezza davvero notevoli, grazie ai numerosi enjambements, alle frequenti sinalefi e all'uso sostanzialmente paratattico della sintassi $^{6}$. E a proposito della paratassi, osserva Mutterle (1977: 21):

Non sarà inopportuno ricordare che l'impiego della paratassi, così generalizzato nel primo Pavese, è tutt'altro indice di primitività e di contatto spontaneo, «realistico», con le cose; al contrario, è complementare alla condizione di assenza del protagonista dilaniato da una doppia personalità, adulta e infantile, borghese e contadina. [...] A chi si sente estraneo nel mondo, la realtà si presenta secondo sequenze disarticolate, che hanno smarrito una precisa gerarchia, e si dispongono secondo un allineamento assurdo ${ }^{7}$.

Insomma: il darsi fenomenico delle cose, «in una lingua che rende mitica e circolare qualsiasi combinazione», si proietta, sì, su un asse sincronico - o acronico - in cui «i nessi temporali e spaziali riescono sommersi dall'equivalenza similarità-contiguità» (Mutterle 1977: 72-73), ma pure si disarticola in momenti antitetici cui solo la casualità del reale e la potenza del 'ritmo stilisti$\mathrm{co}^{8}$ sanno dare un senso e uno scopo.

\section{2.}

La prima situazione oppositiva si trova proprio nell' incipit della lirica, con quel «Ma» che sembra voler riprendere un discorso interrotto, ma non in senso contestuale, bensì esistenziale ${ }^{9}$. E questa situazione oppositiva si continua subito, nel primo verso, grazie al chiasmo tra «notte ventosa» e «limpida notte», dove però bisogna pur sottolineare il virtuale rapporto di causa ed effetto tra

5. Tutta l'opera di Pavese è ricca di coppie antitetiche: città campagna, infanzia maturità, uomo - donna, ozio lavoro, ecc.

6. "Il linguaggio è soggetto a una sintassi, a una coerenza grammaticale, insomma a una tradizione - come i suoni a rapporti matematici, le pietre a esigenze di gravità, e i colori a rapporti cromatici» (Cesare PAVESE, Il mestiere di vivere (Diario 1935-1950), VII ed., Torino: Einaudi, 1965, p. 324).

7. Del resto, come nota Venturi (op. cit., p. 9), le costruzioni paratattiche già caratterizzavano I mari del sud e contribuivano a determinare «una disposizione lineare tale da soddisfare l'esigenza di un verso lungo, prosastico e narrativo".

8. Cfr. Venturi, op. cit., p. 23.

9. Do solo qualche esempio di incipit con $M a$ nella poesia novecentesca: Eugenio Montale, "Ma dove cercare la tomba» (in Ma dove cercare la tomba, da Ossi di seppia); Mario LuzI, «Ma tu continua e perditi, mia vita" (in (se musica è la donna amata), da Avvento notturno); Corrado Govoni, "Ma chi è, chi è che suona» (in Il piano da Fuochi d'artificio; Mario MoretTI, «Ma che è che vaga nell'aria?», (in Che è che vaga nell' aria, da Poesie scritte col lapis, Nino Oxilia, "Ma voi non vedeste la lampa», (in Il saluto ai poeti crepuscolari, da Gli orti). 
«ventosa» e «limpida» (la notte è limpida perché ventosa). E' evidente come Pavese rovesci - oltre a semplificare sintatticamente - la descrizione iniziale de La sera del dì di festa di Leopardi («Dolce e chiara è la notte e senza vento») ${ }^{10}$.

Il «Ma» iniziale e il suddetto chiasmo dànno vita a tutta una serie di opposizioni. Ma giova prima mettere in evidenza gli altri numerosi chiasmi presenti nel testo:

$$
\begin{aligned}
& \text { "notte ventosa» — «limpida notte» } \\
& \text { "calma stupita» - «remoto stupore» } \\
& \text { "calma stupita» - «immobile calma» } \\
& \text { "colli / freschi e neri» — «vaga e limpida immobilità» } \\
& \text { «immobile luce» — «limpida immobilità» } \\
& \text { «immobile luce» — «luce stupita» } \\
& \text { "le foglie [...] stormivano» - «apparivano i colli» } \\
& \text { «immobile calma» - «vivere assorto» } \\
& \text { «immobile calma» - «luce stupita» }
\end{aligned}
$$

Come è evidente, questi chiasmi sono formati fondamentalmente da sostantivi e aggettivi. E non sfugga, infine, come i costituenti del chiasmo fra «immobile luce» e "limpida immobilità" diano vita a due belle, ancorché "tautologiche», sinestesie.

Veniamo così alle opposizioni. Queste concernono tutta una serie di 'campi semantici' che si possono così rappresentare:
(a) luce - buio
(b) stasi - movimento
(c) vita - morte
(d) passato - presente
(e) lontano - vicino
(f) presenza - assenza
(g) astratto concreto
(h) vedere - udire

Ad esempio, per quanto riguarda le opposizioni relative alla luce e al buio, ossia (a), esse chiamano in causa, da un lato, termini come "luce», "giorno", «limpida», «nitide», «estate», e, dall'altro, termini come «buio», «notte», «neri». Per quanto concerne (b), possiamo vedere una contrapposizione fra «immobilità», «immobile», «calma», da una parte, e «vento», «ventosa», «sfiorava», «ritorna», dall'altra. E' ovvio che è possibile incrociare o sovrapporre questi campi semantici oppositivi (con eventuali risvolti metaforici o connotativi). Ad esempio, «luce» può rientrare sia nel campo oppositivo luce - buio, sia nel campo oppositivo vita - morte; oppure «remoto» o «ricordo»/«ricordare» possono far parte tanto del campo oppositivo passato - presente, quanto del campo oppositivo lontano - vicino. Per quanto concerne (d), cioè l'opposizione passato -

10. E’ facilmente verificabile come La notte sia ricca di rinvii (linguistici e tematici) a Leopardi. 
presente, qui il ruolo determinante è svolto dai tempi verbali (imperfetto presente).

Alle opposizioni fanno eco i parallelismi «calma stupita» — «luce stupita», «nell'immobile luce del giorno» — «nell'immobile calma del giorno», «limpida immobilità» — «limpida notte». A questi parallelismi si possono poi affiancare le dittologie "di foglie e di nulla», "freschi e neri» (con assonanza), "vaga e limpida», "nitide e morte».

\section{3.}

La lirica è attraversata, però, anche da tutta una serie di ripetizioni e corrispondenze lessicali, strutturali (più esattamente sintagmatiche) e fonico-timbriche che creano una fitta trama coesiva. Così annotava Pavese (1965: 53), il 9 novembre 1937:

La ripetizione nelle nuove poesie non ha una ragione musicale ma costruttiva. Osservare come le frasi-chiave in esse sono sempre al presente, e le altre vi convergono anche se al passato. Voglio dire che mi succede in queste poesie di afferrare una realtà attuale, non narrativa ma evocativa, dove accade qualcosa a un'immagine, accade ora, in quanto l'immagine viene ora elaborata dal pensiero e veduta agire e affondare le sue radici nella realtà.

La parola o la frase ripetuta non è altro che il nerbo di questa immagine, costruito da cima a fondo come un'impalcatura, il perno per cui la fantasia gira su se stessa e si sostiene appunto come un giroscopio che esiste solo nel presente, in azione, e poi cade e diventa un ferro qualunque ${ }^{11}$.

Vediamo, dunque, i principali fenomeni iterativi. Quanto alle ripetizioni lessicalii ${ }^{12}$, diamo l'elenco completo ${ }^{13}$ :

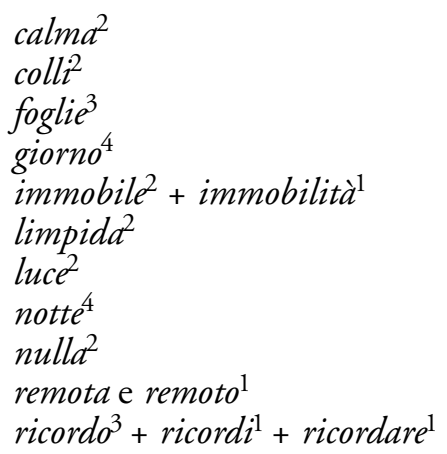

11. I corsivi sono di Pavese.

12. Accanto alle ripetizioni lessicali troviamo le 'accumulazioni' lessicali (cfr. vv. 13-16), che ci ricordano non tanto le 'congerie' petrarchesche quanto piuttosto le 'enumerazioni' di W. Whitman (e appunto di enumerations whitmaniane parla Allen Ginsberg in A Supermarket in California).

13. I lessemi vengono riportati in ordine alfabetico e col numero delle occorrenze in esponente. 


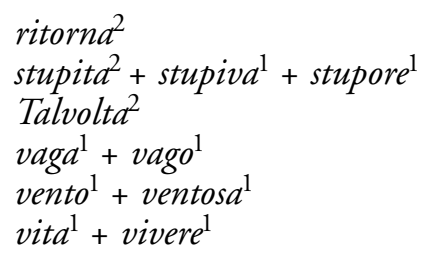

Alle ripetizioni lessicali si affiancano quelle sintagmatiche (o strutturali), che possono essere identiche o simili. Ripetizioni sintagmatiche identiche sono: «di foglie e di nulla» (vv. 4 e 16) e «Talvolta ritorna» (vv. 6 e 16, in posizione anaforica; si può quindi notare come il v. 16 sia costituito quasi interamente da ripetizioni). Ripetizioni sintagmatiche simili sono:

«calma stupita» — «luce stupita» (vv. 3 e 18)

«nell'immobile luce del giorno» — «nell'immobile calma del giorno» (vv. 7 e 17) «luce del giorno» — «cose del giorno» — «calma del giorno» (vv. 7, 13 e 17).

Per quanto concerne le corrispondenze fonico-timbriche, esse sono costituite soprattutto da assonanze, ma non mancano consonanze e allitterazioni:

(i) assonanze:

«vent $O s A »-« \operatorname{rem} O \mathrm{t} A$ »

«fr $E \operatorname{sch} I »-« \mathrm{n} E \mathrm{r} I »$

«vEnt $O »-$ «ci $E l O »$

«perd $U \mathrm{t} A »-« \mathrm{n} U \mathrm{ll} A »$

«c $A \operatorname{lm} A »-$ « $A \mathrm{tt} A$ »

«rem $O t A »-\langle\operatorname{talv} O \mathrm{lt} A »-\langle\operatorname{rit} O \operatorname{rn} A »$

«giOrn $O$ » - «ric $O \operatorname{rd} O$ » - «ass $O \operatorname{rt} O$ » - «rem $O t O »$

«d $O v E »$ - «c $O s E »$ - «c Ost $E »$ - «m Ort $E »$

«v $I$ gn $E »-« \mathrm{n} I \mathrm{t} I \mathrm{~d} E »$

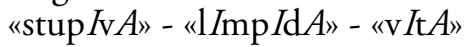

«Ess $A$ » «r Est $A$ »

«trov $A \mathrm{rl} I »-$ «ammass $A \mathrm{t} I »$

(ii) consonanze:

«no TTe» - «tu TTe»

«rito $R N \mathrm{a}$ - «gio $R N \mathrm{No} »$

«piaNTe» - «veNTo»

(iii) allitterazioni:

«notte ventosa»

«Talvolta ritorna nel giorno»

«nell'immobile luce»

«limpida immobilità».

E' anche interessante notare che quest' ultima allitterazione costituisce una sinestesia formata da un aggettivo sdrucciolo e da un sostantivo tronco. 
4.

La notte ripropone, ancora una volta, una delle situazioni pavesiane più tipiche, ossia la contemplazione del mondo attraverso la finestra ${ }^{14}$, ovvero lo scoprire la vita con stupore ${ }^{15}$. Ha precisato Basile (1974: 507):

Questa «finestra-quadro» sulla vita ritrova [...], nella terza lassa della lirica $L a$ notte, un protagonista inedito e una dimensione metafisica aperta al fascino notturno, alla domanda senza risposte [...]. L'immagine acquisisce una notevole complessità: il protagonista è delineato (il bambino), avviene uno scambio tra vuoto dell'interno e vuoto incorniciato dalla finestra, tra oscurità interiore e tenebre esterne che velano il paesaggio tradizionale e vitale (foglie, colli, piante, vigne), la scomparsa della luce guida alla percezione del nulla contrastata dalle consuete immagini di evasione aerea (vita era un'altra ... vento ... cielo).

Se quest' interpretazione è corretta, come riteniamo che sia, allora può aiutarci a spiegare o giustificare le caratteristiche stilistiche, linguistiche e strutturali sopra evidenziate (e cioè la massiccia presenza nella lirica di iterazioni, parallelismi e opposizioni).

Con La notte siamo sicuramente di fronte a un atteggiamento leopardiano, ma fors'anche - e magari solo inconsciamente - post-pascoliano. Ora, non si tratta tanto di insistere ancora una volta sulle immagini simboliche o metaforiche della finestra (spazio e occasione per un atteggiamento voyeuristico) e della collina (sineddoche-simbolo della mammella-donna) già esaustivamente studiate dalla critica ${ }^{16}$, quanto piuttosto di accertare eventuali innovazioni nella rappresentazione di queste immagini.

La finestra è senz’altro la "«finestra-quadro" sulla vita», come rilevato da Basile (1974: 507). Ma... Ma va considerata almeno da una triplice prospettiva: (a) quella del bambino-personaggio che "guardava la notte sui colli», (b) quella del poeta che descrive il paesaggio e si identifica col bambino, e (g) quella del lettore che si identifica col bambino e col poeta. In un certo senso, per usare la terminologia di Genette (1972), (a) e (b) corrispondono, rispettivamente, al 'modo' e alla 'voce' narrativi.

Solo tenendo conto di questa complessità prospettica è possibile afferrare il sottile gioco delle opposizioni e delle ripetizioni. Se le ripetizioni stanno a significare o suggerire una concezione ciclica, immutabile e dunque mitica del tempo ${ }^{17}$,

14. Tralasciando le opere in prosa (soprattutto Paesi tuoi o i racconti Il gruppo e Il Capitano), perlomeno si vedano, in Lavorare stanca, le poesie Il figlio della vedova, Mania di solitudine, Rivelazione, Mattino, Il paradiso sui tetti.

15. "La poesia nasce [...] dagli istanti in cui leviamo il capo e scopriamo con stupore la vita», come annota PAVESE alla data 16 aprile 1940 (Il mestiere di vivere, op. cit., p. 174).

16. Cfr., per es., Fernandez, op. cit. e Bruno Basile, «La 'Finestra' di Pavese. Analisi di un'immagine ossessiva", Lingua e Stile, IX, 3, 1974, pp. 503-518. Cfr. anche Claude Mauron, Dalle metafore ossessive al mito personale, Milano: Il Saggiatore, 1996 e Anco Marzio Mutterle, Limmagine arguta. Lingua, stile, retorica di Pavese, Torino: Einaudi, 1977.

17. Cfr. Bàrberi Seuarotti, op. cit, p. 170; Guglielminetti, op. cit., p. 27; Francesco De Napoli, F., Del mito, del simbolo e d'altro. Cesare Pavese e il suo tempo, Cassino: Garigliano, 2000. 
le opposizioni dei campi semantici sopra evidenziati innescano una complessa strategia metaforico-simbolica.

Infatti, la finestra non è solo il varco, la proiezione, l'apertura verso l'esterno, verso l'ignoto, verso l'oggetto del desiderio, né è solo metafora del ricordo e magia del ricordare i ricordi, ma è essa stessa realizzazione sublimata del desiderio. La finestra è lo spazio attraverso cui osservare e contemplare il mondo, ma è anche parte di quel mondo. La finestra è il poetare attraverso il vedere e il vedere attraverso la poesia, è poesia mediante osservazione e osservazione mediante poesia, è visione poetante e poesia contemplante. Del resto, vedere non significa solo 'vedere-che(-cosa)' (visione puramente fisico-fenomenica), ma anche 'vedere-in' (visione creativo-analogica) e 'vedere-come' (visione storico-culturale) ${ }^{18}$.

La finestra consente la verifica dell'esserci degli oggetti lì di fronte ('oggetto' nel senso di obiectum, Gegenstand) e provoca il ricordo, la fantasia, il desiderio. Per cui lo stupore è non solo né tanto quello del bambino-poeta, ma è soprattutto quello del lettore che viene così a identificarsi sia col bambinopersonaggio, sia col poeta-bambino. Ma...

$M a$ la finestra è anche il vuoto ${ }^{19}$ che lascia penetrare la «notte remota», il ricordo della vita, anzi «il ricordo / di quel vivere assorto» (si noti l'enjambe$m e n t)$. La finestra è simbolo dell'esserci che suggerisce il non-esserci-più e che permette di contemplare la notte che, sfiorata - come una donna o un seno — soltanto dal ricordo, è già, ciclicamente, ricordo, è remota, è morta: è ricordo del ricordo, è quel nulla di fronte a cui la luce del giorno può solo stupirsi. E allora la finestra - come quella nel quadro La Condition humaine di Magritte - è un'illusione su lontananze illusorie ${ }^{20}$. E' un'illusione come la poesia, che, sola, può e sa dire «quel tempo di là dai ricordi».

\section{5.}

L'analisi qui proposta è di matrice sostanzialmente strutturalista. Si discosta però dallo strutturalismo - perlomeno da certo strutturalismo ${ }^{21}$ — in quan-

18. Cfr. Joseph Margolis, “'Vedere-in', 'finzione’, 'trasfigurazione’. La percezione della rappresentazione pittorica”, Discipline Filosofiche, XV, 2, 2005, pp. 43-58. Proprio sul 'vedere-in' e sul 'vedere-come' è basato, per es., il racconto di E. Hemingway Hills Like White Elephants (Cfr. Luciano Vitacolonna, Divagazioni testuali. Studi di testologia semiotica, Lanciano: Carabba Editore, 2004, pp. 163-267).

19. Questo vuoto potrebbe essere simbolizzato - o quantomeno connotato - dagli accapo interni, che creano, appunto, dei vuoti nel testo.

20. Cfr. Antonio Prete, Trattato della lontananza, Torino: Bollati Boringhieri, 2008, pp. 112-113.

21. Mi riferisco, ad es., a Roman Jаковson Poetry of Grammar and Grammar of Poetry (= Selected Writing, vol. 3), edited, with a Preface, by S. Rudy, The Hague-Paris-New York: Mouton, 1981, i cui limiti sono stati ben evidenziati e criticati per es. da Paul Werth «Roman Jakobson's Verbal Analysis of Poetry», Journal of Linguistics, 12, 1976, pp. 21-73 e Janos S. PETÖFI, «I parallelismi di Jakobson dalla prospettiva di una teoria testuale semiotica», Lingua e Stile, XXI, 2-3, 1986, pp. 397-426. Cfr. anche Gerard Genetre, Figures III, Paris: Seuil, 1972. 
to non si limita a individuare e mettere in luce solo le caratteristiche formali (ossia le peculiarità relative al piano dell'espressione), come ad es. i parallelismi, le opposizioni, le ripetizioni, ecc., bensì cerca di indagare anche la dimensione semantica e simbolica della lirica attraverso la funzionalizzazione o pertinentizzazione di quelle caratteristiche.

Da tutto ciò scaturisce una serie di osservazioni sia di natura generale sul ruolo del significante (fonico, metrico, ritmico, grafico, ecc,), sia di natura specifica sul linguaggio di Pavese. Se, come abbiamo detto più sopra, La notte si allontana notevolmente dalla 'lirica prosastica' più tipicamente pavesiana, se ne allontana non perché la poesia diventi 'canto puro' assoluto, ma perché $\mathrm{i}$ temi, le immagini, i simboli necessitano di modalità espressive che, da un lato, riescano a suggerire una concezione ciclica e mitica del tempo, e, dall'altro, sappiano assicurare a quelle tematiche e a quelle immagini una dimensione e un valore universali. E' per questo che La notte, più che ad altre poesie di Pavese, va forse accostata - a nostro parere - non solo a certe pagine dei Dialoghi con Leucò o di La casa in collina, ma anche a certe soluzioni linguistico-formali del Pavese traduttore. 